\title{
Evidence-Based Nutritional Advice for Patients Affected by Age-Related Macular Degeneration
}

\author{
Walter Andreatta Samer El-Sherbiny \\ Birmingham and Midland Eye Centre, Sandwell and West Birmingham Hospitals NHS Trust, Birmingham, UK
}

\section{Key Words}

Age-related macular degeneration · Vision · Nutrition

\begin{abstract}
This paper presents the evidence available in the literature on the role of nutrients in preventing the occurrence of age-related macular degeneration (AMD) and its progression to more advanced stages. In our analysis we considered publications on vitamins $B, C, E$ and D, carotenoids (i.e. lutein, zeaxanthin and $\beta$-carotene), $\Omega-3$ polyunsaturated fatty acids and zinc published between 2003 and 2013. While the evidence supporting supplementation and higher dietary intake of nutrients for AMD prevention is weak to moderate, large and robust randomised controlled trials showed that the AREDS formula leads to a $25 \%$ reduction in progression to advanced AMD in individuals belonging to AREDS categories 3 and 4. After reviewing the current literature, which includes the AREDS2 study, we suggest an 'evidence-based formula'.
\end{abstract}

(c) 2014 S. Karger AG, Basel

\section{Introduction}

Age-related macular degeneration (AMD) is the principal cause of visual loss in older adults in the developed world [1]. In the UK alone, 15,000 people annually are registered as blind or partially sighted because of AMD
$[2,3]$. This accounts for nearly half of all registrations, causing a major social and economic burden.

Particularly in the atrophic form of AMD, limited interventions are available to prevent it or delay its progression. Studies report conflicting data on the role of diet. The health care professional has the duty to advise the patient appropriately about which nutrients and supplements could be beneficial.

In this review, we considered the most commonly used and extensively studied nutrients including vitamins B, C, $\mathrm{E}$ and $\mathrm{D}$, carotenoids (i.e. lutein, zeaxanthin and $\beta$-carotene), $\Omega-3$ polyunsaturated fatty acids (PUFAs) and zinc.

\section{Methods}

We searched the sources Medline, Trip Database, PubMed and the Cochrane library. We reviewed the papers published between 2003 and 2013. We considered the references of the articles extracted to find further relevant publications. In our analysis we appraised large observational and epidemiological studies as well as well-constructed randomised controlled trials (RCTs). Our PICO question was as follows: $\mathrm{P}$ (patient problem) = AMD; I (intervention) $=$ antioxidant/diet/diet supplements/nutrition; $\mathrm{C}$ (comparison) $=$ placebo (or left blank); O (outcome) = reduction in disease progression/risk of disease onset. In addition, we considered national and international guidelines and statements on the role of nutrients in AMD, including those published by the Royal College of Ophthalmologists (UK). The quality of the evidence varies depending on the nutrient discussed.

\section{KARGER}

E-Mail karger@karger.com

www.karger.com/oph (c) 2014 S. Karger AG, Basel

0030-3755/14/2314-0185\$39.50/0
Walter Andreatta, MD, MSc

Birmingham and Midland Eye Centre Dudley Road

Birmingham, B18 7QH (UK)

E-Mail andreattawalter@hotmail.com 


\section{How Can Diet Affect AMD?}

The accumulation of reactive molecules and free radicals is known to cause cell damage in the human body and promote inflammation. This is particularly significant in the eye because of prolonged sunlight exposure (photooxidative stress) and the high oxygen demand of the retina. Oxidative stress and inflammation have been shown by several in vitro and in vivo studies to contribute to AMD formation and progression [4]. Various nutrients including carotenoids (i.e. lutein, zeaxanthin and $\beta$ carotene), vitamins B, C, E and D and PUFAs possess antioxidant and anti-inflammatory effects [5].

PUFAs are present in high content in the retina, and in particular in the photoreceptor outer segments. These are susceptible to oxidation and require constant replenishment. The most studied PUFAs are $\Omega-3$.

Finally, zinc is involved in cell division and cell growth. It was postulated that this mineral helps preventing the degeneration of Bruch's membrane in AMD [6].

\section{Can Diet Prevent AMD Occurrence?}

The strongest evidence on the use of supplements in AMD prevention is provided by large cancer and cardiovascular disease trials where an eye examination was added.

A Cochrane review article published in 2012 looking at the effect of vitamin $\mathrm{C}$, vitamin $\mathrm{E}$, carotenoids (including lutein and zeaxanthin), selenium and zinc found no evidence from RCTs supporting the intake of these nutrients to prevent or delay the onset of AMD. The authors concluded that further large studies are required to assess the safety and effectiveness of these supplements [7]. The main studies included were the Alpha-Tocopherol and Beta-Carotene Study, the Physicians' Health Study 1, the Vitamin E Cataract and ARM Trial and the Women's Health Study [8-11].

The Cochrane review did not include studies considering the role of folic acid, vitamin $\mathrm{B}_{6}$ (pyridoxine hydrochloride) and vitamin $\mathrm{B}_{12}$ (cyanocobalamin) which was assessed in the Women's Antioxidant and Folic Acid Cardiovascular Study [12]. This was a large randomised trial completed in the USA which looked at the effect of the combination of the above nutrients $(2.5 \mathrm{mg} /$ day of folic acid, $50 \mathrm{mg}$ /day of vitamin $B_{6}$ and $1 \mathrm{mg} /$ day of vitamin $B_{12}$ ) in preventing cardiovascular events in women who suffered previous cardiovascular events or had significant risk factors for it. While the nutrients did not reduce cardiovascular disease, they decreased the risk of developing
AMD by $35-40 \%$ after a mean treatment and follow-up period of 7.3 years.

Concerning $\Omega-3$ PUFAs, a Cochrane review published in 2012 could not find high-quality RCTs assessing the role of the nutrients in AMD prevention [13]. Nevertheless, epidemiological studies suggested that the intake of food with a high concentration of $\Omega-3$ PUFAs could decrease the risk of AMD [14-16]. For example, a large population-based cohort study in Australia revealed that 1 serving of fish per week, and similarly 1 or 2 servings of nuts per week, was linked to a reduced risk of early AMD 10 years later. A higher intake was not associated with an increased protection [16]. One serving corresponds to $145 \mathrm{~g}$.

There are no well-conceived RCTs exploring the role of vitamin D in AMD. However, the NHANES 3, a large cross-sectional study involving 7,752 individuals, discovered that participants over 40 years of age consuming daily milk and/or fish at least once weekly had a reduced risk of early AMD and soft drusen compared to those who consumed these products less than weekly and less than twice a month, respectively [17]. However, the presence of $\Omega-3$ PUFAs in fish could have confounded the results.

In summary, some cohort studies have found an association between the intake of some nutrients and the prevention of AMD development (table 1). However, no large and well-constructed RCT has shown that healthy individuals would benefit from supplements or high dietary intake of specific nutrients in order to prevent AMD development.

\section{Can Diet Prevent AMD Progression?}

The Age-Related Eye Disease Study (AREDS) and AREDS2 are the significantly largest and most robust RCTs investigating the role of nutrients in AMD progression $[18,19]$.

The AREDS is a multicentre RCT which ran between 1995 and 2001 in the USA. In the analysis 3,640 participants aged 55-80 years were included. AMD grading was divided into 4 categories according to increasing severity [18]:

- category 1: a few small drusen or no drusen;

- category 2: pigment abnormalities, extensive small drusen or at least 1 intermediate size druse;

- category 3: geographic atrophy not involving the centre of the macula, extensive intermediate drusen or at least 1 large druse;

- category 4: advanced AMD or visual acuity less than 20/32 due to AMD in 1 eye. 
Table 1. Level of evidence on the use of supplements in preventing AMD

\begin{tabular}{|c|c|c|c|}
\hline Factor & Strong & Moderate & Weak \\
\hline Lutein & $\mathrm{X}$ & & \\
\hline Zeaxanthin & $\mathrm{X}$ & & \\
\hline $\bar{\beta}$-Carotene & $\mathrm{X}$ & & \\
\hline Vitamin C & $\mathrm{X}$ & & \\
\hline Vitamin E & $\mathrm{X}$ & & \\
\hline Zinc & $\mathrm{X}$ & & \\
\hline Folic acid & & $\sqrt{ } 2.5 \mathrm{mg} /$ day supplements ${ }^{\mathrm{a}}$ & \\
\hline Vitamins $\mathrm{B}_{6} / \mathrm{B}_{12}$ & & $\sqrt{50} \mathrm{mg} / 1 \mathrm{mg} /$ day supplements ${ }^{\mathrm{a}}$ & \\
\hline$\Omega-3$ PUFAs & & & $\begin{array}{l}\sqrt{ } \text { Fish weekly ( } 1 \text { serving) } \\
\sqrt{ } \text { Nuts weekly }(1-2 \text { servings) }\end{array}$ \\
\hline Vitamin D & & & $\begin{array}{l}\sqrt{\text { Milk once daily }} \\
\sqrt{ } \text { Fish once per week }\end{array}$ \\
\hline
\end{tabular}

$\mathrm{X}$ indicates the presence of evidence against the nutrient, $\sqrt{ } \mathrm{B}_{6} / \mathrm{B}_{12}$, PUFAs and vitamin $\mathrm{D}$ mean evidence supporting their use $\sqrt{;} 1$ serving $=145 \mathrm{~g}$. ${ }^{\text {a }}$ Only in women with previous cardiovascular events or multiple cardiovascular risk factors.

Subjects were randomised to the intervention arms listed below:

- antioxidants: vitamin C (500 mg) + vitamin E (400 IU) $+\beta$-carotene $(15 \mathrm{mg})$

- zinc (80 mg) + copper (2 mg) to prevent anaemia;

- antioxidants + zinc and copper;

- placebo.

The risk of progression was assessed at the final follow-up after a mean period of 6.3 years.

Taking into consideration only categories 3 and 4, the group randomised to antioxidants + zinc and copper was $25 \%$ less likely to progress to advanced AMD compared to placebo. No effect in the use of supplements was found in early AMD (categories 1 and 2).

Therefore the authors concluded that antioxidants plus zinc should be taken in category 3 and 4 patients older than 55 who do not smoke.

A limitation of the study was the inclusion of mainly Caucasian individuals who were healthier and had a higher level of education compared to the average population. In addition, the effect of supplementation was relatively modest, and no suggestion was made on how long the patients should be treated for.

An observational follow-up study on the AREDS participants 5 years after the conclusion of the RCT showed that the beneficial effect of the antioxidants + zinc combination persisted [20].

No statistically significant serious events occurred revealing an excellent safety profile of these combinations.

The Role of Nutrients in Preventing

Occurrence and Progression of AMD
Nevertheless, studies using similar doses of $\beta$-carotene found a higher risk of developing lung cancer in smokers $[8,21]$. In addition, the dose of zinc used in the study was believed by many researchers to be excessive and was shown in the AREDS to lead to increased hospital admission secondary to urinary tract infections.

Moreover, over the following years, some observational and small RCTs advocated a beneficial role of higher dietary intake of the carotenoids lutein and zeaxanthin as well as $\Omega-3$ PUFAs such as eicosapentaenoic acid (EPA) and docosahexaenoic acid (DHA) [14-16, 22, 23].

Therefore a new study named AREDS2 was commissioned to investigate the role of these nutrients in preventing AMD progression which was published in May 2013 [19]. This was a large multicentre RCT based in the USA which ran between 2006 and 2012 and recruited 4,203 participants aged 50-85 fulfilling the criteria of AREDS category 3-4.

Individuals were randomised to the following arms:

- AREDS formula;

- AREDS formula + lutein (10 mg/day $)+$ zeaxanthin ( $2 \mathrm{mg} /$ day);

- AREDS formula + DHA (350 mg) + EPA (650 mg);

- AREDS formula + lutein $(10 \mathrm{mg} /$ day $)+$ zeaxanthin (2 mg/day) and DHA (350 mg) + EPA (650 mg).

The subjects then underwent secondary randomisation to investigate the effect of the elimination of $\beta$-carotene and the reduction of the dose of zinc to $25 \mathrm{mg} /$ day in the AREDS formula. 
Primary randomisation revealed that the above carotenoid and PUFA supplements had no statistically significant effect on AMD progression. Subgroup analysis showed no effect in the use of PUFAs. However, there was a protective effect in subjects taking lutein and zeaxanthin compared to those individuals in the lowest quintile of their dietary intake. In addition, post hoc analysis revealed a $10 \%$ reduction in the progression to advanced AMD in patients taking the AREDS formulation with lutein and zeaxanthin compared to those who had the AREDS nutrients without $\beta$-carotene.

There was no decreased efficacy or reduction in vision because of lower zinc dosage or $\beta$-carotene exclusion from the AREDS formula. Finally, the incidence of lung cancer $(2 \%)$ in the group randomised to $\beta$-carotene was significantly increased. Most of these individuals were exsmokers. No current smokers were allocated to the $\beta$-carotene group in the study.

Similar to AREDS, one of the limitations of AREDS2 was the inclusion of mostly highly educated and well-nourished Caucasian individuals. In addition, the study design was complex, including primary and secondary randomisation. Finally, no placebo group was included in the study.

In summary, the AREDS formulation should be used with the lower zinc dose and no $\beta$-carotene, particularly in current and former smokers. The publication reporting the AREDS2 results advised that further studies investigating the role of lutein and zeaxanthin are required. Nevertheless, the AREDS2 author Prof. Paul Bernstein presenting the study at the Royal College of Ophthalmologists Congress in 2013 (Liverpool, UK) advocated that it was reasonable, in the AREDS combination with a lower zinc dosage, to substitute $\beta$-carotene with lutein and zeaxanthin.

A Cochrane review was published in 2012 on the role of nutrients in AMD progression including vitamin C, vitamin $\mathrm{E}$, carotenoids (i.e. lutein, zeaxanthin and $\beta$ carotene), selenium and zinc [24]. The authors concluded that the only large, appropriately powered trial with a long follow-up period was AREDS and embraced its recommendations.

Regarding PUFAs, a Cochrane review published in early 2013 could not find strong evidence to support their use and advocated the need for further high-quality studies on this topic such as the AREDS2 which was not yet published at the time [13]. AREDS2 found no effect of $\Omega-3$ PUFAs in preventing AMD progression even in the subgroup analysis.

However, the Nutritional AMD Treatment 2 Study, a well-constructed RCT published in 2013, found that PUFAs can prevent progression to exudative AMD [25].
Participants of this study were randomised either to oral supplementation of $840 \mathrm{mg} /$ day DHA and $270 \mathrm{mg} /$ day EPA or to placebo. In addition, EPA and DHA levels in the red blood cell membranes were measured. Although no effect was found when considering the entire cohort, subgroup analysis revealed that subjects in the highest tertile of EPA/DHA concentration in the red blood cell membranes had a $68 \%$ risk reduction in developing exudative AMD over 3 years.

The disparity with the findings of the AREDS2 could be explained by the different inclusion criteria and PUFA dosages, the dissimilar methods of subgroup analysis but also by the lack of a placebo group in the AREDS2. The use of AREDS nutrients in every group might have led to a ceiling effect, masking the potential benefits of fatty acids.

Finally, there is no good evidence supporting the intake of vitamin $\mathrm{D}$ to delay or prevent AMD progression.

In view of the latest RCTs and Cochrane reviews, we propose the use of the following combination in AREDS category 3 or 4 patients. We call this evidence-based (EB) formula (table 2):

- vitamin C (500 mg) + vitamin E (400 IU);

- $\operatorname{zinc}(25 \mathrm{mg})+\operatorname{copper}(2 \mathrm{mg} /$ day $)$.

In addition, lutein $(10 \mathrm{mg} /$ day $)$ and zeaxanthin $(2 \mathrm{mg} /$ day) are likely to have a positive effect if used instead of $\beta$-carotene (table 2).

However, health care practitioners should make the patients aware of the possibility of unknown long-term side effects of the supplements (over 10 years for zinc, copper, vitamin $\mathrm{C}$ and $\mathrm{E}$, and over 5 years for lutein and zeaxanthin). In addition, the evidence to support lutein and zeaxanthin is not as strong as for the other supplements.

\section{Where Are These Nutrients Found?}

The dietary intake of the nutrients discussed should be part of a healthy and varied diet (table 3). Despite this, it is sometimes difficult to reach the daily amounts advised by AREDS and AREDS2. For example, as reported by the Department of Agriculture in the USA, an individual should eat every day either half a cup of cooked kale, or a cup of cooked spinach, or a cup and a half of cooked broccoli in order to reach $10 \mathrm{mg}$ of lutein intake (box 1). Therefore, the use of supplements is a convenient and generally cost-effective option.

Regarding AMD prevention, various formulations exist on the market containing folic acid, vitamins $\mathrm{B}_{6}$ and $\mathrm{B}_{12}$. Unfortunately we could not find a single product with all these nutrients in doses similar to those reported 
Table 2. Level of evidence on the use of supplements in preventing AMD progression

\begin{tabular}{llll}
\hline Factor & Strong & Moderate & Weak \\
\hline EB formula & $\sqrt{ }$ & & \\
EB formula with lutein + zeaxanthin & & $\sqrt{ }$ \\
$\Omega-3$ PUFAs & & & $\sqrt{ }$ \\
\hline
\end{tabular}

$\sqrt{ }$ indicates the presence of evidence supporting the intake of the nutrients.

$\mathrm{EB}=$ Evidence-based
Table 3. Dietary products with the highest concentrations of the listed nutrients

\begin{tabular}{ll}
\hline $\begin{array}{l}\text { Lutein and } \\
\text { zeaxanthin }\end{array}$ & Green leafy vegetables (i.e. spinach and kale) \\
\hline$\Omega-3$ & $\begin{array}{l}\text { Eggs, fish (salmon, tuna, herring, mackerel and sardines), nuts, } \\
\text { flaxseeds, pumpkin seeds and soybeans }\end{array}$ \\
\hline Vitamin C & $\begin{array}{l}\text { Fruits (i.e. orange, lemon, mango, papaya, pineapple, strawberries, } \\
\text { blueberries, watermelon) } \\
\text { Vegetables (i.e. broccoli, cauliflower, peppers, leafy greens, } \\
\text { potatoes, tomatoes) }\end{array}$ \\
\hline Vitamin E & Wheat germs and sunflower seeds \\
\hline Vitamin D & Fatty fish, fish oil, milk, eggs and certain mushrooms \\
\hline Zinc & Meat and vegetables such as nuts, whole grains and legumes \\
\hline
\end{tabular}

in table 1. Patients could take preparations providing each nutrient individually.

However, the health care professional should clearly make the patient aware that the evidence to support their use is moderate and only applies to women with previous cardiovascular events or multiple cardiovascular risk factors.

Concerning the progression to advanced AMD, we conducted an online research to find which products on the market currently contain the EB formula with lutein and zeaxanthin. Unfortunately, none of the reviewed formulations complied with the exact combination and dosage. However, some adhered more than others, and new products are continuously brought onto the market.

As authors we are not in a position to recommend any specific brand. Nevertheless, our paper provides an updated literature review which can be used by health care professionals to help their patients to choose the most evidence-based preparation available in their area.

Several studies demonstrated an increased macular pigment density following diet supplementation with various nutrients [26, 27]. Objective techniques such as fundus reflectance and autoreflectance, and subjective devices like the heterochromatic flicker photometer have been investigated and could be employed in the fu-
Box 1. Information for patients.

- AMD Alliance: http://www.amdalliance.org/ amdalliance/AMDAlliance/Home

- Royal National Institute of Blind People: http://www.rnib.org.uk/eyehealth/eyeconditions/ conditionsac/Pages/amd.aspx

- Macular Society: http://www.macularsociety.org

- NHS UK: http://www.nhs.uk/Conditions/ Macular-degeneration/Pages/Introduction.aspx

- United States Department for Agriculture: http://www.nel.gov/

ture to detect the most suitable candidates for nutritional supplementation and monitor their response to treatment.

\section{Disclosure Statement}

We declare the following interests: none. 


\section{References}

$>1$ Congdon N, O'Colmain B, Klaver CC, et al, the Eye Diseases Prevalence Research Group: Causes and prevalence of visual impairment among adults in the United States. Arch Ophthalmol 2004;122:477-485.

$>2$ Klein R, Klein BEK, Linton KL: Prevalence of age-related maculopathy. The Beaver Dam Eye Study. Ophthalmology 1992;99:933-943.

3 Evans JR, Rooney C, Dattani N, et al: Causes of blindness and partial sight in England and Wales. Health Trends 1996;28:5-12.

$\checkmark 4$ Khandhadia S, Lotery A: Oxidation and agerelated macular degeneration: insights from molecular biology. Expert Rev Mol Med 2010 12:e34.

5 Christen WG, Glynn RJ, Hennekens CH: Antioxidants and age-related eye disease. Current and future perspectives. Ann Epidemiol 1996;6:60-66.

6 Julien S, Biesemeier A, Kokkinou D, et al: Zinc deficiency leads to lipofuscin accumulation in the retinal pigment epithelium of pigmented rats. PLoS One 2011;6:e29245.

7 Evans JR, Lawrenson JG: Antioxidant vitamin and mineral supplements for preventing agerelated macular degeneration. Cochrane Database Syst Rev 2012;6:CD000253.

$\checkmark 8$ The Alpha-Tocopherol, Beta Carotene Cancer Prevention Study Group: The effect of vitamin $\mathrm{E}$ and beta carotene on the incidence of lung cancer and other cancers in male smokers. N Engl J Med 1994;330:1029-1035.

$>9$ Hennekens $\mathrm{CH}$ : A randomized trial of aspirin and beta-carotene among US physicians. Prev Med 1985;14:165-168.

10 Taylor HR, Tikellis G, Robman LD, et al: Vitamin E supplementation and age-related maculopathy. Invest Ophthalmol Vis Sci 2001;42:311.

-11 Buring JE, Hennekens CH: Randomized trials of primary prevention of cardiovascular disease in women. An investigator's view. Ann Epidemiol 1994;2:111-114.
12 Christen WG, Glynn R, Chew EY, et al: Folic acid, pyridoxine, and cyanocobalamin combination treatment and age-related macular degeneration in women. The Women's Antioxidant and Folic Acid Cardiovascular Study. Arch Intern Med 2009;169:335-341.

13 Lawrenson JG, Evans JR: Omega 3 fatty acids for preventing or slowing the progression of age-related macular degeneration. Cochrane Database Syst Rev 2012;11:CD010015.

14 Chong EW, Robman LD, Simpson JA, et al: Fat consumption and its association with agerelated macular degeneration. Arch Ophthalmol 2009; 127:674-680.

15 Christen WG, Schaumberg DA, Glynn RJ, et al: Dietary omega-3 fatty acid and fish intake and incident age-related macular degeneration in women. Arch Ophthalmol 2011;129: 921-929.

16 Tan JS, Wang JJ, Flood V, Mitchell P: Dietary fatty acids and the 10-year incidence of agerelated macular degeneration: the Blue Mountains Eye Study. Arch Ophthalmol 2009; 127:656-665.

17 Parekh N, Chappell RJ, Millen AE, et al: Association between vitamin D and age-related macular degeneration in the Third National Health and Nutrition Examination Survey, 1988 through 1994. Arch Ophthalmol 2007; 125:661-669.

18 Age-Related Eye Disease Study Research Group: Report 8. Arch Ophthalmol 2001;119: 1417-1436.

19 Age-Related Eye Disease Study 2 Research Group: Lutein + zeaxanthin and omega-3 fatty acids for age-related macular degeneration: the Age-Related Eye Disease Study 2 (AREDS2) randomized clinical trial. JAMA 2013;309:2005-2015.
20 Chew EY, Clemons TE, Agrón E, et al: Longterm effects of vitamins $\mathrm{C}$ and $\mathrm{E}, \beta$-carotene, and zinc on age-related macular degeneration: AREDS report No 35. Ophthalmology 2013;120:1604-1611.

-21 Omenn GS, Goodman GE, Thornquist MD, et al: Effects of a combination of beta carotene and vitamin A on lung cancer and cardiovascular disease. N Engl J Med 1996;334:11501155.

22 Tan JSL, Wang JJ, Flood V, Mitchel Pl: Dietary fatty acids and the 10-year incidence of age-related macular degeneration: the Blue Mountain Eye Study. Arch Ophthalmol 2009; 127:656-665.

23 Seddon JK, et al: Dietary carotenoids, vitamins $\mathrm{A}, \mathrm{C}$, and $\mathrm{E}$, and advanced age-related macular degeneration. Eye Disease CaseControl Study Group. JAMA 1995;272:14131420 .

24 Evans JR, Lawrenson JG: Antioxidant vitamin and mineral supplements for slowing the progression of age-related macular degeneration. Cochrane Database Syst Rev 2012;11: CD000254.

25 Souied EH, Delcourt C, Querques G, et al: Oral docosahexaenoic acid in the prevention of exudative age-related macular degeneration: the Nutritional AMD Treatment 2 Study. Ophthalmology 2013;120:1619-1631.

26 Bernstein PS, Ahmed F, Liu A, et al: Macular pigment imaging in AREDS2 participants: an ancillary study of AREDS2 subjects enrolled at the Moran Eye Center. Invest Ophthalmol Vis Sci 2012;53:6178-6186.

27 Bartlett H, Howells O, Eperjesi F: The role of macular pigment assessment in clinical practice: a review. Clin Exp Optom 2010;93:300308 